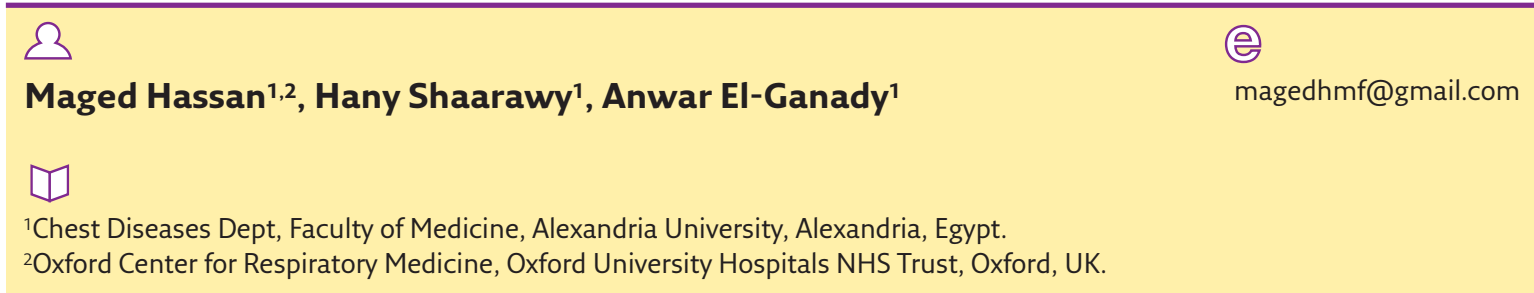

2Oxford Center for Respiratory Medicine, Oxford University Hospitals NHS Trust, Oxford, UK.

\title{
Respiratory training in Egypt
}

In Egypt, respiratory medicine (more commonly termed "chest diseases") is regarded as a separate speciality rather than a subspecialty of internal medicine. All graduates of Egyptian Medical Schools have to complete a year of internship at teaching hospitals rotating between different medical and surgical departments. Following internship and obtaining licence to practice, doctors are required to work for 6-12 months at primary healthcare facilities around the country. At the end of this period, a doctor can start their specialty training; the choice of which depends on the cumulative score they achieved at medical school.

The chest diseases specialty has not often been among the very popular choices but this trend is seeing some change. Respiratory training is offered either through teaching (university) hospitals or Ministry of Health ( $\mathrm{MOH})$ hospitals. Respiratory training at $\mathrm{MOH}$ is offered most usually at chest hospitals with a few places at chest departments in general hospitals. Recruitment to university hospitals is a very competitive process because of the perceived better training. The duration of the training programme (termed residency in Egypt) is 4-5 years. Alongside training, residents are expected to pursue postgraduate studies either by applying for a place in a Master's Programme in chest disease at one of the medical schools [1] or in the Pulmonology Fellowship Programme [2] (run by the $\mathrm{MOH}$ ). The Master's Programme involves attending lectures on different respiratory topics, clinical teaching and preparing a research thesis. The Pulmonology Fellowship Programme involves both theoretical and clinical teaching but without research. Under both programmes, candidates are required to pass several examinations after each course. In addition, residents not training at the central chest hospital in Cairo (where the Pulmonology Fellowship Programme takes place) or at one of the teaching hospitals (which run the Master's Programme), are expected to do a 1-year clinical visitorship at either of the aforementioned institutions depending on the postgraduate programme pursued. Given that the Egyptian fellowship is based in Cairo, signing up for Master's degree is the more popular choice outside the capital.
Residency in chest diseases is one of the most daunting jobs in a hospital because residents are expected to manage a busy ward and a respiratory high-dependency unit (HDU), and be on call for the emergency department and referrals from other clinical departments in a hospital. Despite that, it is a very exciting speciality because of the range of procedures available to learn, both in acute medicine situations and for chronic cases.

\section{Inpatient experience}

In a typical shift, the team of chest residents is in charge of the inpatient ward, the chest HDU (with an on-call specialist) and consultations from different departments. During the first year, the priorities of the residents are to acquire the skills to manage these duties well. Junior residents are trained to perform pleural procedures, mainly pleural aspiration and chest drain insertion. In the past few years, the use of ultrasound has gained ground in terms of availability and practice in respiratory practice in Egypt. It is commonplace now that residents use thoracic ultrasound to perform pleural tapping safely, which is a very common cause for consultation for respiratory residents by internal medicine departments because of the high prevalence of chronic hepatitis C infection [3] and hepatic hydrothorax in Egypt. Management of common cases in the chest diseases ward (exacerbations of asthma and chronic obstructive pulmonary disease, and pleuropulmonary infections) is among the core parts of training for residents. The other important skills new residents are trained in are advanced management of airways, advanced life support, invasive and noninvasive mechanical ventilation, and cardiorespiratory monitoring; this is because most chest departments have their own HDUs caring for patients with respiratory failure and severe infections. Consultations in the general medical ward, other than for thoracentesis, are usually left for more senior residents who have better knowledge of extrapulmonary links with chest diseases.
Cite as: Hassan $\mathrm{M}$, Shaarawy H, El-Ganady A. Respiratory training in Egypt. Breathe 2017; 13: 247-249. 


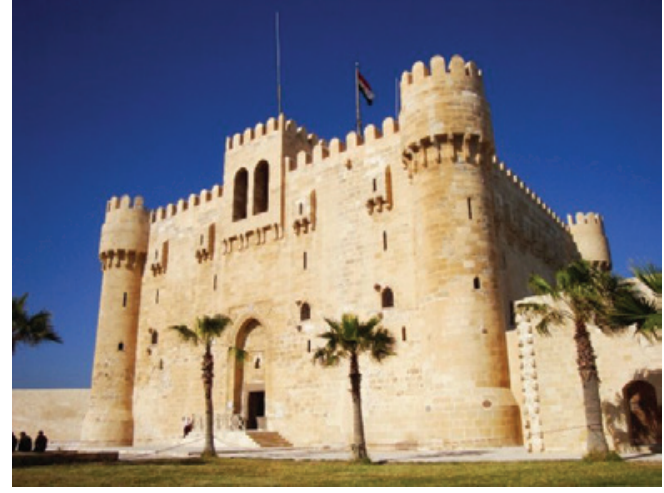

Qaitbay Fortress in Alexandria (courtesy of Dr Mohamed Gouda).

\section{Outpatients}

Residents are also expected to run outpatient clinics, either alone or along with specialist clinics. Very few centres have subspecialty clinics (like interstitial lung disease, cancer, etc.). The only exception to this are tuberculosis clinics, which are an integral part of chest hospitals (affiliated with $\mathrm{MOH}$ ), and these are the only places that are allowed to isolate patients with pulmonary tuberculosis or dispense outpatient treatments for patients with noncommunicable disease. For this reason, residents working for chest hospitals benefit from extensive training in the management of tuberculosis.

\section{Procedures}

Apart from thoracentesis and chest tube insertion, more senior residents, in their second and third years of training, are allowed to be trained in other advanced procedures. Flexible bronchoscopy is now available in all teaching hospitals and in almost all chest hospitals, and at the end of the residency, trainees are expected to perform diagnostic bronchoscopy competently. Medical thoracoscopy is still emerging, and is currently available in some teaching hospitals and a few chest hospitals. Interventional bronchoscopy is available in a handful of teaching hospitals only and is not part of the routine training during residency. Sleep medicine is gaining popularity and polysomnography is being offered in most teaching hospitals now, which exposes residents to the practicalities of polysomnography as well as the management of patients with sleep disordered breathing.

\section{Teaching and supervision}

Both the Master's and Fellowship programmes are structured to provide theoretical and clinical knowledge of respiratory diseases. In the theoretical parts, residents are required to attend various courses covering knowledge of different aspects of the speciality. Clinical teaching is also regularly organised alongside the lectures. Both theoretical and clinical examinations are held at the end of each academic semester. This process is essential in gaining knowledge about the management of different conditions, as well as the use and interpretation of pulmonary function tests, radiological studies and other investigations.

Residents working at teaching hospitals and large general hospitals practise under supervision of professors and consultants and clinical rounds on wards are held regularly. Residents are also trained in endoscopic procedures under supervision of consultants and specialists performing procedures. For residents working at smaller hospitals, this is achieved during their 1-year visitorship.

Most departments have weekly scientific meetings to discuss all interesting and difficult cases in a multidisciplinary fashion involving consultants from cardiothoracic surgery, oncology and radiology. A journal club is frequently organised during these meeting and trainees are asked to prepare talks about recent respiratory research. A yearly conference is organised by most academic departments, which is useful for trainees to update their knowledge and meet professors from different universities.

\section{Research}

Undergoing research is an essential part of training for residents taking the Master's pathway (rather than the purely clinical Pulmonology Fellowship). At the end of their residency, and in order to obtain the Master's degree, a resident is required to not only have passed the theoretical and clinical examinations but also to have taken part in research conducted at the chest department of the university to which they are attached. Given that the Master's Programme is the more widely pursued pathway, most residents undertake research at some point of their residency.

\section{Challenges}

The main problem for respiratory training in Egypt is that it is not homogeneous across the different institutions. The quality of training acquired at teaching hospitals and central chest hospitals is usually better because of the range and complexity of cases referred to such tertiary healthcare institutions, the wider array of investigative tools available, and the close supervision by consultants and professors. For trainees in other hospitals, the visiting resident system has depended for a long time on the personal motivation of each doctor, which is not a guarantee that quality training has been attained. In recent years, a monitoring system has been put in place where log books are required to be signed off for all essential competencies and is now a requirement, besides passing examinations, for completion of training and becoming a specialist in chest diseases [4]. It is hoped this system will improve training quality nationwide. 


\section{Conflict of interest}

None declared.

\section{References}

1. Alexandria Faculty of Medicine. Postgraduate Programs. www.med.alexu.edu.eg/postgraduate-programs-2/ Date last accessed: May 5, 2017

2. Egyptian Fellowship Board. Pulmonology Fellowship Training Program. www.egyfellow.mohealth.gov.eg/ Fileupload/\%d8\%a7\%d9\%84\%d8\%b2\%d9\%85\%d8\%a7\% d9\%84\%d8\%a9\%20\%20\%d8\%a7\%d9\%84\%d9\%85\%d8 \%b5\%d8\%b1\%d9\%8a\%d8\%a9\%20\%d9\%84\%d9\%84\%d 8\%a3\%d9\%85\%d8\%b1\%d8\%a7\%d8\%b6\%20\%d8\%a7\%d 9\%84\%d8\%b5\%d8\%af\%d8\%b1\%d9\%8a\%d8\%a9.pdf Date last accessed: May 5, 2017.

3. Mohamoud YA, Mumtaz GR, Riome S, et al. The epidemiology of hepatitis $C$ virus in Egypt: a systematic review and data synthesis. BMC Infect Dis 2013; 13: 288.

4. Alexandria Faculty of Medicine. Master Degree of Chest Diseases. www.med.alexu.edu.eg/wp-content/ uploads/2017/05/Chest.pdf Date last accessed: May 5, 2017. 